ISSN 0103-9954

\title{
AGRUPAMENTO DE ÁRVORES MATRIZES DE Eucalyptus grandis EM FUNÇÃO DAS VARIÁVEIS DENDROMÉTRICAS E DAS CARACTERISTICAS TECNOLÓGICAS DA MADEIRA
}

\section{GROUPING OF Eucalyptus grandis SELECTED TREES IN FUNCTION OF THE DENDROMETRIC VARIABLES AND THE TECHNOLOGICAL CHARACTERISTICS OF WOOD}

\author{
Merielen de Carvalho Lopes ${ }^{1}$ Clovis Roberto Haselein ${ }^{2}$ Elio José Santini ${ }^{3}$ \\ Solon Jonas Longhi ${ }^{4}$ Silviana Rosso ${ }^{5} \quad$ Denis L. G. Fernandes ${ }^{5} \quad$ Leonel Freitas de Menezes ${ }^{6}$ \\ RESUMO
}

O objetivo deste trabalho foi investigar a possibilidade de agrupar árvores matrizes de Eucalyptus grandis Hill ex Maiden em função das variáveis dendrométricas e fenotípicas e das características tecnológicas da madeira. As 63 árvores matrizes selecionadas, em um povoamento comercial do litoral norte do estado do Rio Grande do Sul foram abatidas e classificadas quanto às características fenotípicas e dendrométricas. Depois disso, retiraram-se discos na base, no diâmetro à altura do peito (DAP), e a 25, 50, 75 e $100 \%$ da altura comercial, destinados à determinação da massa específica básica. Das duas primeiras toras seccionadas acima do DAP, foram retiradas, paralelamente à medula, as tábuas destinadas à avaliação qualitativa da madeira serrada. Por meio da análise de agrupamento, as árvores matrizes foram reunidas em quatro grupos distintos e classificadas com base na tendência em desenvolver rachaduras de topo nas tábuas e toras. No grupo I, classificaram-se as árvores com menor tendência a apresentar rachaduras de topo das tábuas, enquanto que nos grupos II e III, reuniram-se as árvores com menor tendência a rachaduras de topo das toras; e por fim, no grupo IV, classificaram-se as árvores com maior tendência a rachaduras de topo das toras e das tábuas. Através da aplicação da análise discriminante, foi possível identificar cinco variáveis com poder de discriminação dos grupos (DAP, porcentagem estimada de rachaduras, relação altura/diâmetro, tipo de casca e percentual volumétrico de alburno) e três funções discriminantes. A técnica de análise de agrupamento e análise discriminante possibilitou o agrupamento das árvores semelhantes, a identificação das variáveis de seleção e a discriminação das funções que permitem alocar novas árvores nos grupos formados.

Palavras-chave: análise de agrupamento; Eucalyptus grandis; dendrometria; tecnologia da madeira.

\section{ABSTRACT}

The objetive of this study is to cluster selected trees of Eucalyptus grandis Hill ex-Maiden as a function of phenotypic, dendrometric and wood technology characteristics. In a 27 years old commercial stand, located in the northern coast of Rio Grande do Sul, 63 selected trees were cut down and classified to the phenotypic and dendrometric characteristics. Then, disks were taken at the base, 25, 50, 75 and 100\% of the commercial height, and at the $\mathrm{DBH}$, for basic density determination. From the two first logs above the DBH boards parallel to the pith were taken, with $25 \mathrm{~mm}$ of thickness, for sawn wood qualitative evaluation. The selected trees were grouped into four distinct groups following cluster analysis. In Group I were classified the trees with a lower tendency of presenting boards with end splits while in Groups II and III were classified the trees with a lower tendency of presenting logs with end splits and, in Group IV were classified the trees with a higher tendency of presenting end splits of the boards and the logs. For these

1. Engenheira Florestal, MSc., Bolsista CNPq/DTI, SENAI - Centro Tecnológico do Mobiliário, Rua Hans Dieter Schmidt, 879, CEP 89290-000, São Bento do Sul (SC). meire@senai-sc.ind.br

2. Engenheiro Florestal, PhD., Professor Adjunto do Departamento de Ciências Florestais, Centro de Ciências Rurais, Universidade Federal de Santa Maria, CEP 97105-900, Santa Maria (RS). haseleic@ccr.ufsm.br

3. Engenheiro Florestal, Dr., Professor Adjunto do Departamento de Ciências Florestais, Centro de Ciências Rurais, Universidade Federal de Santa Maria, CEP 97105-900, Santa Maria (RS). santinie@ccr.ufsm.br

4. Engenheiro Florestal, Dr., Professor Titular do Departamento de Ciências Florestais, Centro de Ciências Rurais, Universidade Federal de Santa Maria, CEP 97105-900, Santa Maria (RS). longhiso@ccr.ufsm.br

5. Acadêmico do Curso de Engenharia Florestal, Centro de Ciências Rurais, Universidade Federal de Santa Maria, CEP 97105-900, Santa Maria (RS).

6. Engenheiro Florestal, International Business in Eucalyptus (IBE), Av. Dr. Salvador França, 889/401, CEP 90690000, Porto Alegre (RS). fmleonel@terra.com.br

Recebido para publicação em 15/09/2003 e aceito em 4/06/2004. 
groups, by discriminant analysis, there were identified five variables with real power of discrimination (DBH, estimated log split perimeter, ratio height/diameter (rh/d), type of bark and sapwood volumetric percentage) and three discriminant functions. The technique of the multivariate analyze was an efficient method of grouping the selected trees of Eucalyptus grandis. The discriminant analysis enabled the selection of variables with real power of sorting the trees and determinating the discriminant functions that allow the allocation of future trees in the cluster.

Key words: grouping analysis; Eucalyptus grandis; dentometric characteristics; wood technology.

\section{INTRODUÇÃO}

O Brasil destaca-se no plantio das espécies do gênero Eucalyptus devido à adaptabilidade destas às condições edafo-climáticas brasileiras, a facilidade para propagação vegetativa e ao rápido crescimento. Porém, a madeira de eucalipto apresenta algumas características que dificultam sua melhor utilização na indústria da madeira e em mobiliários. Tais características, como tendência a rachaduras, empenamentos, nós, entre outras, podem ser revertidas com o uso de técnicas de melhoramento, através da identificação dos caracteres desejáveis e da aplicação do manejo florestal correto.

A variabilidade dentro e entre as espécies de eucalipto introduzidas no país promove a base para a investigação dos programas de melhoramento florestal. A alta variabilidade dentro das espécies de eucaliptos (Zobel e Jett, 1995) constitui a base dos estudos para propagação e qualificação de árvores com características superiores e específicas ao uso industrial. A análise da madeira para serraria, realizada em várias espécies e híbridos de eucalipto, demonstra altos níveis de variabilidade para a maioria das características. Isso possibilita a seleção de árvores com propriedades tecnológicas superiores à média da população (Assis, 2000).

A técnica de análise multivariada é uma ferramenta amplamente empregada nas análises estruturais de florestas, principalmente para estudos fitossociológicos. Entretanto, alguns pesquisadores vêm aplicando essas técnicas para seleção de árvores com base nas propriedades físico-mecânicas da madeira.

Através do uso de técnicas de análise multivariada em progênies de meios irmãos de Eucalyptus grandis, Xavier et al. (1996) concluíram que os conceitos e aplicações proporcionam uma ferramenta a mais na seleção final das famílias que irão compor um pomar de sementes por mudas ou ainda na formação de pomares clonais.

A partir de grupos baseados em características botânicas e silviculturais, Ruy et al. (2001) constatarm que os clones superiores de E. urophylla podem ser agrupados pelas características de qualidade da madeira (massa específica básica, comprimento e diâmetro dos lume das fibras) e que conjuntamente, os parâmetros de qualidade da madeira e botânicos/silviculturais podem ser usados para ensaios de seleção de clones superiores.

Araújo (2002) aplicou a análise multivariada com a finalidade de agrupar espécies amazônicas similares em termos de propriedades físico-mecânicas. Para o autor, a massa específica da madeira seria o melhor indicativo para usos dependentes da resistência a esforços mecânicos.

Louzada et al. (2002), através da utilização de técnicas de análise multivariada, identificaram 3 agrupamentos por comportamento de secagem da madeira, ou seja, os autores utilizaram espécies comerciais portuguesas com o intuito de agrupar aquelas com características similares na secagem da madeira.

Santos et al. (2003), através do uso de progênies de Eucalyptus grandis, avaliaram que a massa específica, a resistência à compressão paralela e a resistência à flexão estática mostraram-se propriedades promissoras para fins de melhoramento, enquanto que para a resistência ao cisalhamento paralelo, não foi detectada variabilidade genética na população estudada.

Com vistas a encontrar material compatível com os padrões produtivos e qualitativos da indústria madeireira, o presente estudo teve como objetivo principal agrupar árvores matrizes de Eucalyptus grandis Hill ex Maiden em função das variáveis dendrométricas e das características tecnológicas da madeira. 


\section{MATERIAL E MÉTODOS}

Para aplicação da análise de agrupamento, foram selecionadas 63 árvores matrizes de Eucalyptus grandis Hill ex Maiden em um povoamento comercial com 27 anos, localizado no litoral norte do Estado do Rio Grande do Sul, pertencentes à Flosul Indústria e Comércio de Madeiras Ltda. A Tabela 1 apresenta os valores médios das variáveis dendrométricas e tecnológicas analisadas, cuja forma de determinação desses dados pode ser encontrada em Lopes (2003). Quanto às características fenotípicas (não incluídas na Tabela 1), as árvores utilizadas neste trabalho podem ser classificadas, em sua maioria, como perfeitamente retas, copa grande e rala composta por galhos abertos e grossos, casca lisa e resíduo rugoso.

TABELA 1: Média e coeficiente de variação das variáveis dendrométricas e tecnológicas analisadas.

TABLE 1: Average and variation coefficient of dentrometric and technological variables analyzed.

\begin{tabular}{|c|c|c|}
\hline $\begin{array}{r}\text { Propriedades avaliadas } \\
\end{array}$ & Média & Coeficiente de Variação (\%) \\
\hline Diâmetro à altura do peito $(\mathrm{cm})$ & 66,4 & 10,7 \\
\hline Altura comercial (m) & 37,5 & 6,24 \\
\hline Altura total $(\mathrm{m})$ & 49,8 & 5,01 \\
\hline Percentual volumétrico de cerne (\%) & 75,6 & 6,60 \\
\hline Percentual volumétrico de alburno (\%) & 24,4 & 20,5 \\
\hline Percentual volumétrico de casca (\%) & 3,45 & 19,8 \\
\hline Volume comercial com casca $\left(\mathrm{m}^{3}\right)$ & 6,63 & 20,0 \\
\hline Volume comercial sem casca $\left(\mathrm{m}^{3}\right)$ & 6,44 & 20,1 \\
\hline Massa específica básica $\left(\mathrm{g} / \mathrm{cm}^{3}\right)$ & 0,46 & 8,78 \\
\hline Massa seca (kg) & 3064 & 21,8 \\
\hline Conicidade $(\mathrm{cm} / \mathrm{m})$ & 1,41 & 15,7 \\
\hline Fator de forma artificial & 0,51 & 8,00 \\
\hline Relação altura/diâmetro & 1,33 & 11,6 \\
\hline Porcentagem estimada de rachaduras das toras (\%) & 0,47 & 55,8 \\
\hline Rachaduras de topo das tábuas (\%) & 63,5 & 24,4 \\
\hline Encurvamento $(\mathrm{mm} / \mathrm{m})$ & 3,81 & 41,2 \\
\hline Índice de nós por metro quadrado (nós $/ \mathrm{m}^{2}$ ) & 4,88 & 29,2 \\
\hline Diâmetro do maior nó $(\mathrm{cm})$ & 2,79 & 37,0 \\
\hline
\end{tabular}

A classificação das árvores, em função de suas características fenotípicas, dendrométricas e tecnológicas, foi feita através de Análises de Agrupamento, também conhecidas como Técnicas de Classificação que são métodos estatísticos multivariados.

Aplicou-se, com auxílio do Programa SPSS (Statistical Package for the Social Science) duas técnicas de Classificação: Análise de Cluster ou Análise de Agrupamento propriamente dita, que são as técnicas que criam os agrupamentos a partir das informações contidas nos dados e Análise Discriminante, que alocam indivíduos (árvores) a classes previamente estabelecidas.

A análise de agrupamento foi realizada a partir de uma matriz constituída por 63 árvores matrizes, caracterizadas por 27 variáveis. Esta matriz foi padronizada pelo método da variável Z, recomendada por Batista apud Longhi (1997).

A análise de Cluster ou de agrupamento foi realizada utilizando-se o Método Aglomerativo Hierárquico. Este método permite uma série de uniões entre as árvores similares. Começam pela fusão sucessiva das árvores, que se combinam por suas semelhanças, repetindo o procedimento até esgotar as possibilidades de combinação ou até que não fiquem árvores isoladas.

Para permitir a união entre os grupos, foi utilizado o Método Ward de ligação ou Método da Mínima Variância, o qual permite inicialmente calcular a média de todas as variáveis em cada grupo. Após, calculase a distância Euclidiana quadrática, medida de fácil cálculo e amplamente utilizada nas análises de agrupamentos. Este cálculo permite o agrupamento em pares das árvores similares, com base no menor incremento, resultado da soma geral da distância quadrática entre os grupos. 
A representação das seqüências de agrupamentos formados foi apresentada na forma de um dendrograma ou diagrama em árvore. Esta hierarquia resultante expressa um índice de ligação, no qual cada ligação corresponde a um valor numérico, que representa o nível em que têm lugar os agrupamentos. Quanto maior o índice, mais heterogêneas são as árvores agrupadas. Uma vez formado o dendrograma, a separação dos grupos é realizada, observando-se as ramificações formadas num corte em determinado nível, geralmente, na metade da maior distância, embora possa ser feito segundo um critério subjetivo e pessoal.

A análise discriminante foi utilizada com o objetivo de testar diferenças estatísticas significantes em um dado nível de probabilidade, entre os grupos resultantes, determinar funções discriminantes que permitam classificar novas árvores nos grupos, construir regras de alocação para identificar as árvores como membros de um dos $n$ grupos obtidos e estimar a probabilidade de classificações corretas.

Utilizou-se o método STEPWISE, que seleciona as variáveis para entrar na análise, baseando-se nas suas capacidades de discriminação. O processo se inicia, selecionando a variável que apresenta maior valor de discriminação. Esta variável é pareada com as demais variáveis, uma de cada vez, e o critério de seleção é novamente comparado. A variável que, em conjunto com a primeira selecionada, produzir o melhor valor para o critério, é a segunda variável escolhida para entrar na análise. Estas duas variáveis selecionadas são combinadas com as demais remanescentes, e a combinação que apresentar o maior valor para o critério de seleção determinará a terceira variável a entrar na equação. Do mesmo modo, todas as demais variáveis são testadas através do critério de seleção, até que todas sejam ordenadas pelas suas capacidades de discriminação.

O método de seleção utilizado foi o Lambda de Wilks $\left(\Lambda^{*}\right)$, que considera como critério de seleção de variáveis o valor da Estatística $F$ Multivariada, para o teste da diferença entre os centróides dos grupos. A variável que maximiza o valor da estatística $F$, também minimiza o $\Lambda^{*}$, que é uma medida de discriminação entre os grupos.

\section{RESULTADOS E DISCUSSÃO}

\section{Análise de agrupamento de Cluster}

A Figura 1 mostra o dendrograma formado a partir da matriz de dados padronizados, pela Análise de Cluster, utilizando-se a distância euclidiana quadrada como medida de similaridade entre as árvores, através do Método Ward de ligação. O eixo vertical representa a distância euclidiana re-escalonada de 0 a 25 . No eixo horizontal, encontram-se as árvores que geraram os grupos.

Os grupos foram definidos pelo traçado de uma linha paralela ao eixo horizontal, denominada "Linha Fenon". Optou-se pela aplicação da "Linha Fenon", na distância euclidiana de aproximadamente 11, um pouco abaixo da distância média, tendo em vista que um corte na distância média uniria os dois primeiros grupos, estatisticamente distintos, conforme análise posterior. Observa-se, assim, um corte de quatro ramos no agrupamento formado, o que permite a classificação do conjunto das 63 árvores matrizes analisadas em 4 grupos distintos.

\section{Análise discriminante}

Após cinco steps, foram selecionadas cinco variáveis que representam real poder de discriminação dos grupos (Tabela 2). A seleção das variáveis baseou-se nos baixos valores da estatística Lambda de Wilks $\left(\lambda^{*}\right)$. As duas primeiras variáveis, diâmetro à altura do peito (DAP) e percentagem estimada de rachaduras (PER), com valores de $\mathrm{F}$ elevados, foram as mais importantes para caracterizar os grupos, enquanto que as três restantes, tipo de casca (CT), relação altura/diâmetro (RHD) e percentual volumétrico de alburno $\left(\% \mathrm{~V}_{\mathrm{AB}}\right)$, apresentaram valores inferiores, mas que influenciaram na separação dos mesmos. 
Distância Euclidiana Reescalonada

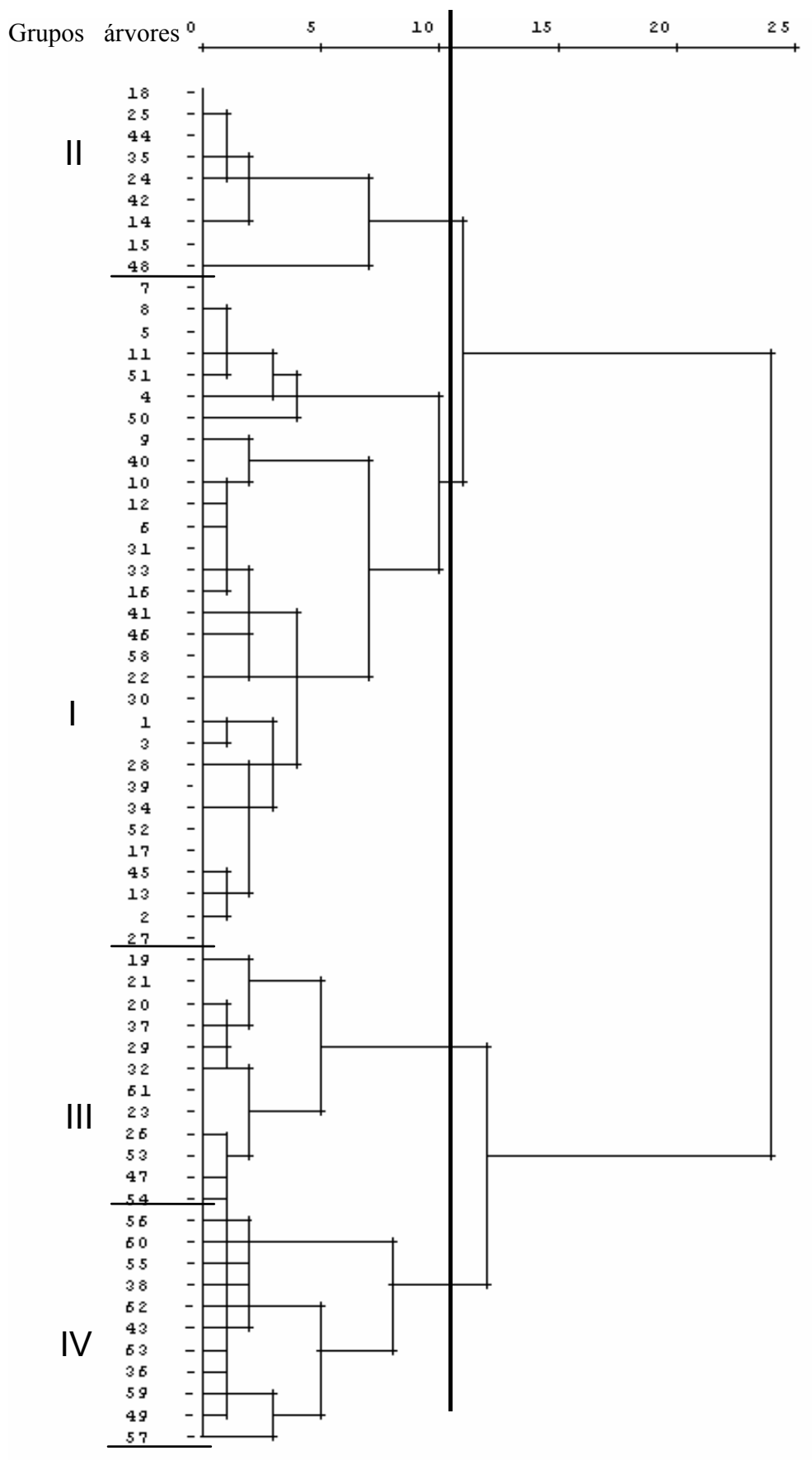

FIGURA 1: Dendrograma com base no método de ligação Ward obtido pela análise de Cluster.

FIGURE 1: Dendrogram based on Ward linking method obtained by cluster analyze. 
TABELA 2: Teste de seleção de variáveis discriminantes, com 5 steps, para a matriz de dados das árvores matrizes de Eucalyptus grandis.

TABLE 2: Test for selection of discriminant variables, with 5 steps, for the data matrix of the Eucalyptus grandis trees.

\begin{tabular}{c|c|c|c}
\hline Step & Variável & F & Lambda de Wilks $\left(\lambda^{*}\right)$ \\
\hline 1 & DAP & 37,684 & - \\
\hline 2 & DAP & 37,428 & 0,500 \\
& PER & 16,039 & 0,343 \\
\hline \multirow{2}{*}{3} & DAP & 38,066 & 0,388 \\
& PER & 19,937 & 0,265 \\
& CT & 8,548 & 0,187 \\
\hline \multirow{2}{*}{4} & DAP & 17,655 & 0,182 \\
& PER & 19,112 & 0,189 \\
& CT & 8,538 & 0,136 \\
& RHD & 7,172 & 0,129 \\
\hline & DAP & 16,554 & 0,146 \\
& PER & 19,730 & 0,159 \\
& CT & 6,870 & 0,105 \\
& RHD & 6,173 & 0,102 \\
& \%V & 4,046 & 0,093 \\
\hline
\end{tabular}

Em que: $\mathrm{DAP}=$ diâmetro à altura do peito; $\mathrm{PER}=$ porcentagem estimada de rachaduras; $\mathrm{CT}=$ tipo de casca; $\mathrm{RHD}=$ relação altura/diâmetro; $\% \mathrm{~V}_{\mathrm{AB}}=$ percentual volumétrico de alburno.

O método pressupõe que valores de $F$ inferiores a 3,84 sejam eliminados da análise, pois possuem baixo poder de discriminação (Longhi, 1997). Desta forma, após 5 steps, obteve-se o valor mínimo de $F$ para discriminação dos grupos e determinação das variáveis com poder de discriminação. Definidas as variáveis discriminantes, realizou-se a determinação das funções discriminantes importantes na análise da influência destas variáveis na formação dos grupos.

Pelo teste estatístico Qui-quadrado $\left(\chi^{2}\right)$ e Lambda de Wilks $\left(\lambda^{*}\right)$, foi possível determinar três funções discriminantes com poder de discriminação. A Tabela 3 apresenta a seleção das funções discriminantes lineares para as variáveis selecionadas.

TABELA 3: Seleção das funções discriminantes para a matriz de dados das árvores matrizes de Eucalyptus grandis.

TABLE 3: Selection of the discriminant functions for the data matrix of the Eucalyptus grandis trees.

\begin{tabular}{l|c|c|c|c|c|c|c|r|r}
\hline Função & Autovalor & $\begin{array}{c}\% \\
\text { da var. }\end{array}$ & $\begin{array}{c}\text { \% var. } \\
\text { acumulada }\end{array}$ & $\begin{array}{c}\text { Correlação } \\
\text { Canônica }\end{array}$ & $\begin{array}{c}\text { Após a } \\
\text { função }\end{array}$ & $\lambda^{*}$ & $\chi^{2}$ & GL & $*$ \\
\hline 1 & 2,409 & 53,2 & 53,2 & 0,841 & $1-2$ & 0,077 & 147,8 & 15 &, 000 \\
2 & 1,695 & 37,4 & 90,7 & 0,793 & $2-3$ & 0,261 & 77,27 & 8 &, 000 \\
3 & 0,423 & 9,3 & 100,0 & 0,545 & - & 0,703 & 20,27 & 3 &, 000 \\
\hline
\end{tabular}

Em que: * = significância a $95 \%$ de probabilidade.

Observa-se que houve a predominância da Função 1 sobre a Função 2 pela análise dos autovalores e que ambas predominaram sobre a Função 3. Para representar $100 \%$ da variância total, foram necessárias as três funções discriminantes, porém a Função 1 representou 53,2\% da variação total, enquanto que a Função 3 representou 9,3\% da variância.

Os valores de correlação canônica foram suficientemente elevados para mostrar o grau de relacionamento entre as funções discriminantes e o grupo de variáveis de maneira satisfatória.

Analisando os coeficientes padronizados das funções discriminantes (Tabela 4), observa-se que houve a predominância da variável DAP e do tipo de casca na primeira função, enquanto que, na segunda função, destacou-se o DAP, o perímetro estimado de rachaduras e o percentual volumétrico de alburno. Na terceira função, a variável relação altura/diâmetro (RHD) se destacou em relação às demais avaliadas. 
TABELA 4: Coeficientes padronizados das funções discriminantes lineares selecionadas.

TABLE 4: Standardized coefficients of the linear discriminant functions selected.

\begin{tabular}{l|c|c|c}
\hline Variáveis & \multicolumn{3}{|c}{ Funções } \\
\cline { 2 - 4 } & 1 & 2 & 3 \\
\hline DAP & 1,084 & 0,902 & $-1,001$ \\
PER & $-0,693$ & 0,639 & 0,325 \\
\%V & $-0,193$ & 0,426 & $-0,409$ \\
RHD & $-0,313$ & $-0,431$ & 1,489 \\
CT & 0,524 & $-0,376$ & $-0,354$ \\
\hline
\end{tabular}

Em que: $\mathrm{DAP}=$ diâmetro à altura do peito; $\mathrm{PER}=$ porcentagem estimada de rachaduras; $\% \mathrm{~V}_{\mathrm{AB}}=$ percentual volumétrico de alburno; $\mathrm{RHD}=$ relação altura/diâmetro; $\mathrm{CT}=$ tipo de casca.

Através dos coeficientes padronizados, pode-se avaliar as variáveis mais importantes na classificação de cada grupo e, por fim, definir as suas respectivas funções discriminantes (Tabela 5).

TABELA 5: Coeficientes da classificação das funções discriminantes para cada grupo.

TABLE 5: Coefficients of the discriminant functions for each group.

\begin{tabular}{|c|c|c|c|c|}
\hline \multirow[t]{2}{*}{ Variáveis } & \multicolumn{4}{|c|}{ Grupos } \\
\hline & I & II & III & IV \\
\hline DAP & $-1,824$ & $-2,065$ & 7,113 & $-0,931$ \\
\hline PER & $-0,431$ & $-0,999$ & $-1,529$ & 3,699 \\
\hline$\% \mathrm{~V}_{\mathrm{AB}}$ & $-0,159$ & $-1,281$ & 0,182 & 1,296 \\
\hline RHD & $-0,00564$ & 3,174 & $-2,717$ & 0,208 \\
\hline $\mathrm{CT}$ & 0,206 & 0,356 & 1,155 & $-2,213$ \\
\hline Constante & $-2,053$ & $-3,237$ & $-5,534$ & $-5,608$ \\
\hline
\end{tabular}

Em que: $\mathrm{DAP}=$ diâmetro à altura do peito; $\mathrm{PER}=$ porcentagem estimada de rachaduras; $\% \mathrm{~V}_{\mathrm{AB}}=$ percentual volumétrico de alburno; RHD = relação altura/diâmetro; $\mathrm{CT}=$ tipo de casca.

Os pontos médios ou centróides de cada grupo, nas três funções discriminantes encontradas, podem ser observados na Tabela 6 e visualizados na Figura 2 do Mapa territorial, que demonstra a distribuição desses pontos para cada grupo.

TABELA 6: Centróides das funções discriminantes obtidos para os quatro grupos das árvores matrizes de Eucalyptus grandis.

TABLE 6: Centroids of the discriminant functions obtained for the four groups of the Eucalyptus grandis trees.

\begin{tabular}{l|c|c|c}
\hline \multirow{2}{*}{ Grupos } & \multicolumn{3}{|c}{ Funções } \\
\cline { 2 - 4 } & 1 & 2 & 3 \\
\hline I & $-0,565$ & $-0,940$ & $-0,364$ \\
II & 0,925 & $-0,909$ & 1,421 \\
III & 2,594 & 1,191 & $-0,385$ \\
IV & $-1,996$ & 2,093 & 0,283 \\
\hline
\end{tabular}




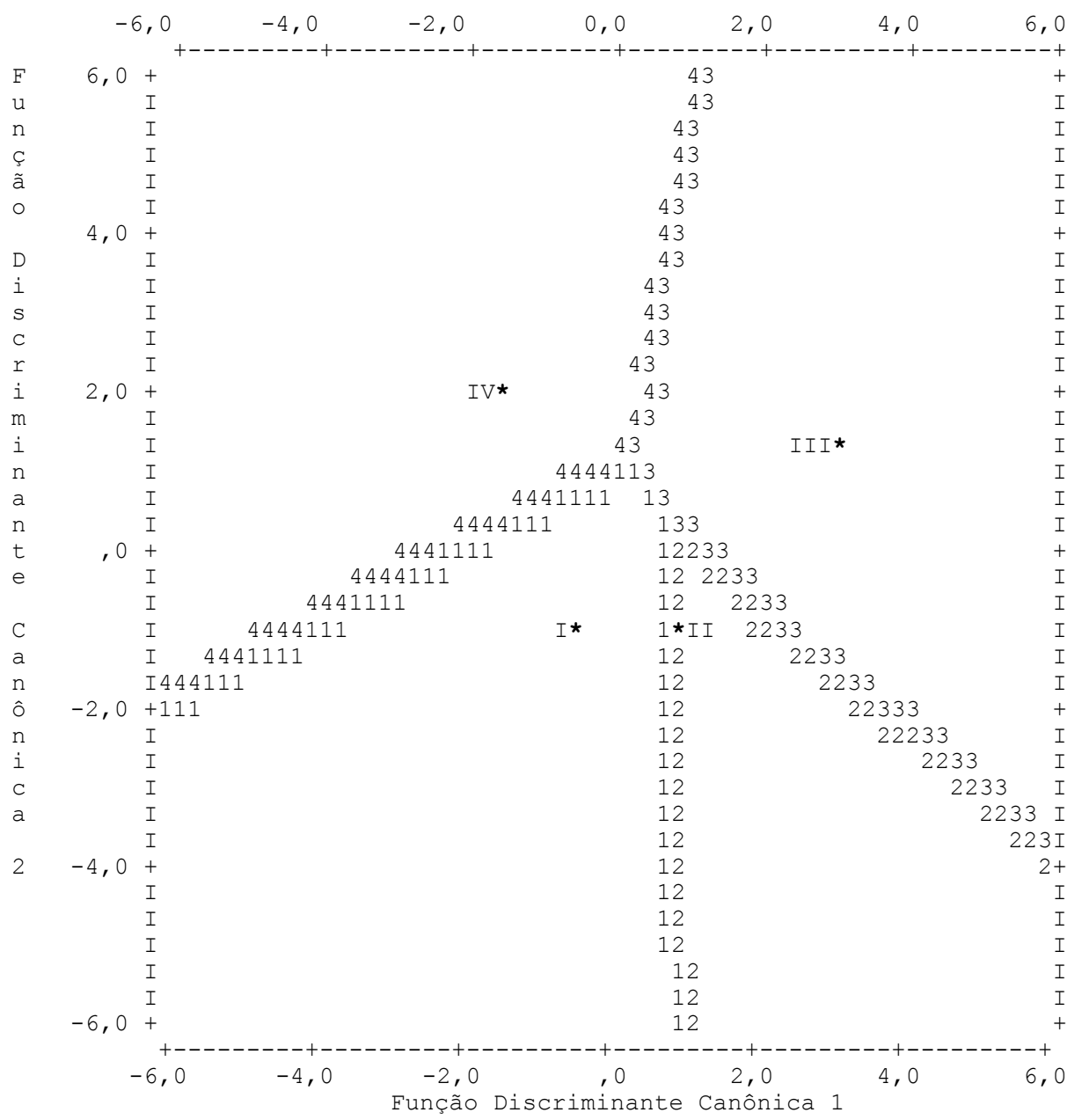

FIGURA 2: Mapa territorial dos grupos determinados, com a localização de seus respectivos centróides. FIGURE 2: Territorial map of the groups determined, with allocation of theirs respective centroids.

$\mathrm{Na}$ Tabela 7, encontram-se os percentuais de classificações corretas e incorretas das árvores estudadas. Com exceção do Grupo III, que não apresentou nenhuma classificação errada, os demais grupos apresentaram erros em suas determinações. No Grupo I, três indivíduos foram mal classificados, dois no Grupo II e um no Grupo III; no Grupo II, dois indivíduos foram classificados erroneamente, um no Grupo I e um no Grupo III; no Grupo IV, duas árvores foram mal classificadas no Grupo II.

TABELA 7: Número de casos e percentagens de classificação das árvores nos quatro grupos obtidos.

TABLE 7: Number of cases and percentages of trees graded in the four groups obtained.

\begin{tabular}{|c|c|c|c|c|c|}
\hline \multirow[t]{2}{*}{ Grupos } & \multirow{2}{*}{$\begin{array}{l}\text { Número de } \\
\text { Árvores }\end{array}$} & \multicolumn{4}{|c|}{ Grupos } \\
\hline & & I & II & III & IV \\
\hline \multirow[t]{2}{*}{ I } & 31 & 28 & 2 & 1 & 0 \\
\hline & $100 \%$ & $90,3 \%$ & $6,5 \%$ & $3,2 \%$ & - \\
\hline \multirow[t]{2}{*}{ II } & 9 & 1 & 7 & 1 & 0 \\
\hline & $100 \%$ & $11,1 \%$ & $77,8 \%$ & $11,1 \%$ & - \\
\hline \multirow[t]{2}{*}{ III } & 12 & 0 & 0 & 12 & 0 \\
\hline & $100 \%$ & - & - & $100 \%$ & - \\
\hline IV & 11 & 2 & 0 & 0 & 9 \\
\hline
\end{tabular}

Em que: $88,9 \%$ das árvores foram classificadas corretamente. 
De modo geral, as três funções discriminantes classificaram corretamente $88,9 \%$ da população estudada, indicando a precisão da técnica de agrupamento. Com isso, elas podem ser usadas para classificar novas árvores entre os grupos.

Para alocação de uma nova árvore em um dos 4 grupos, calculou-se o valor de cada discriminante para essa árvore $\left(y_{1}, y_{2}, y_{3}{ }^{\prime}\right)$, através da Tabela 4, e as distâncias Euclidianas do ponto calculado aos centróides de cada grupo $\left(\mathrm{d}_{1}, \mathrm{~d}_{2}, \mathrm{~d}_{3}, \mathrm{~d}_{4}\right)$ da Tabela 5. A menor distância Euclidiana obtida indicou o grupo em que a nova árvore deveria ser alocada. Dessa forma, uma nova árvore considerada dominante no povoamento pode ser classificada dentro do agrupamento formado, tomando-se as cinco variáveis (DAP, PER, CT, RHD $\left.\mathrm{e} \% \mathrm{~V}_{\mathrm{AB}}\right)$ e os coeficientes das três funções discriminantes.

\section{Classificação geral dos grupos}

Através da análise de Cluster, foram obtidos quatro grupos distintos entre si, que foram caracterizados através dos valores médios das características analisadas, as quais são apresentadas na Tabela 8.

TABELA 8: Médias das variáveis estudadas nos grupos, determinadas pela análise de agrupamento.

TABLE 8: Average values of the variables studied in the groups, determined by the cluster analyze.

\begin{tabular}{lcccc}
\hline Variáveis & Grupo i & Grupo ii & Grupo iii & Grupo iv \\
\hline Número de árvores & 31 & 9 & 12 & 11 \\
\hline $\mathrm{DAP}(\mathrm{cm})$ & 61,7 & 68,8 & 76,9 & 66,3 \\
\hline $\mathrm{HC}(\mathrm{m})$ & 36,9 & 35,4 & 38,7 & 39,1 \\
\hline $\mathrm{HT}(\mathrm{m})$ & 49,8 & 46,9 & 51,2 & 50,8 \\
\hline Relação altura/diâmetro & 1,24 & 1,47 & 1,51 & 1,31 \\
\hline Conicidade $(\mathrm{cm} / \mathrm{m})$ & 1,33 & 1,43 & 1,66 & 1,36 \\
\hline Fator de forma com casca & 0,53 & 0,49 & 0,48 & 0,50 \\
\hline $\mathrm{VC}_{\mathrm{c} / \mathrm{c}}\left(\mathrm{m}^{3}\right)$ & 5,85 & 6,51 & 8,68 & 6,72 \\
\hline $\mathrm{VC}_{\mathrm{s} / \mathrm{c}}\left(\mathrm{m}^{3}\right)$ & 5,68 & 6,32 & 8,42 & 6,52 \\
\hline$\% \mathrm{~V}_{\mathrm{CA}}(\%)$ & 3,45 & 3,28 & 3,46 & 3,6 \\
\hline$\% \mathrm{~V}_{\mathrm{CE}}(\%)$ & 75,9 & 80,4 & 75,1 & 71,8 \\
\hline$\%$ V & 24,1 & 19,6 & 24,9 & 28,2 \\
\hline Massa específica básica $\left(\mathrm{g} / \mathrm{cm}^{3}\right)$ & 0,46 & 0,44 & 0,46 & 0,49 \\
\hline Massa seca (kg) & 2616 & 2806 & 3852 & 3170 \\
\hline Rachaduras de topo das toras $(\%)$ & 0,40 & 0,36 & 0,37 & 0,88 \\
\hline Rachaduras de topo das tábuas $(\%)$ & 57,0 & 70,2 & 66,2 & 73,7 \\
\hline Encurvamento $(\mathrm{mm} / \mathrm{m})$ & 4,24 & 2,84 & 3,77 & 3,42 \\
\hline Índice de nós/ ${ }^{2}$ & 3,14 & 3,30 & 3,05 & 3,16 \\
\hline Diâmetro do maior nó $(\mathrm{cm})$ & 2,70 & 2,59 & 3,10 & 2,90 \\
\hline
\end{tabular}

Em que: $\mathrm{VC}_{\mathrm{c} / \mathrm{c}}=$ volume comercial com casca; $\mathrm{VC}_{\mathrm{s} / \mathrm{c}}=$ volume comercial sem casca; $\% \mathrm{~V}_{\mathrm{CA}}=$ percentual volumétrico de casca; $\% \mathrm{~V}_{\mathrm{CE}}=$ percentual volumétrico de cerne; $\% \mathrm{~V}_{\mathrm{AB}}=$ percentual volumétrico de alburno.

Com base nas características dendrométricas, foi possível determinar que o grupo III está constituído pelas árvores com as maiores médias de DAP, $\mathrm{HC}$ e HT e, conseqüentemente, com os maiores valores de rendimento em volume comercial. Pode-se concluir que este grupo apresenta os melhores critérios de seleção em termos de variáveis dendrométricas. Enquanto isso, o grupo I foi formado pelas árvores com os menores valores médios das características dendrométricas.

Entretanto, os demais grupos não devem ser descartados por apresentarem valores inferiores ao grupo III, pois cada grupo é formado por diferentes árvores. Assim, podem ocorrer exemplares que apresentam boas características dendrométricas, por exemplo a árvore 57 do grupo IV, a qual apresenta volume comercial com casca igual a $7,44 \mathrm{~m}^{3}$.

Pela análise dos dados, foi possível observar uma distribuição uniforme dos valores médios de massa específica básica das árvores entre os grupos. Os grupos I e III apresentaram valores ao redor da 
média geral caracterizada previamente; enquanto que o grupo IV apresentou a maior amplitude e média dos valores de massa específica dentre os grupos. Como exemplo da amplitude dos valores de massa específica, dentro do Grupo IV, destacam-se a árvore $\mathrm{n}^{\mathrm{o}} 55$, com $0,60 \mathrm{~g} / \mathrm{cm}^{3}$, e a árvore $\mathrm{n}^{\circ} 63$, com $0,43 \mathrm{~g} / \mathrm{cm}^{3}$. Já o grupo II apresentou os menores valores médios para a massa específica, porém não difere significativamente dos grupos I e III.

A porcentagem estimada de rachaduras (PER), juntamente com o DAP, foram as duas principais variáveis que influenciaram a classificação dos grupos.

O grupo III destacou-se também por apresentar as menores médias dos valores da porcentagem estimada de rachaduras (PER), formando, em conjunto com o grupo II, os grupos constituídos pelas árvores com a menor tendência a apresentar rachaduras de topo das toras. Observa-se a elevada tendência do grupo IV em apresentar rachaduras de topo das toras, assim desqualificando suas árvores para uma utilização na indústria de madeira serrada, devido às elevadas perdas de material proporcionadas por este defeito.

Baseando-se nas características tecnológicas da madeira serrada, foi possível determinar que o grupo I apresentou a menor tendência a rachaduras de topo das tábuas, porém o grupo III apresenta uma amplitude aceitável de valores das rachaduras de topo das tábuas em relação à média dos demais grupos. Os grupos II e IV apresentam valores médios superiores a $70 \%$ de rachaduras de topo das tábuas, o que indica uma alta propensão a produzir tábuas defeituosas.

De acordo com a classificação, o grupo III apresentou as melhores médias dos valores dendrométricos e pode ser classificado como de baixa tendência em apresentar rachaduras de topo das toras e tábuas. Comparando-se com o grupo I, que apresentou as menores médias de rachaduras de topo das tábuas, porém agrupou as árvores com os menores valores dendrométricos, o grupo III se classifica como o melhor grupo em termos de rendimento e qualidade da madeira serrada.

Entretanto, na concepção de qualidade da madeira serrada, devem ser considerados os defeitos qualitativos avaliados como os empenamentos e nós. Desta forma, o grupo II apresentou os menores valores médios de encurvamento das tábuas, não diferindo dos grupos III e IV, enquanto que o grupo I difere entre os grupos pela média elevada de encurvamento das tábuas.

Pelos critérios qualitativos da madeira, destaca-se novamente o grupo II com a menor amplitude de variação dos valores de índice de nós (INA) e menor média de diâmetro do nó (DMN); porém o menor INA pertenceu ao grupo III, assim como a maior média de DMN entre os grupos. Já os grupos I e IV agruparam as árvores com a maior variação de INA e com os valores intermediários para o DMN entre os demais grupos.

A terceira variável que apresentou poder de discriminação dos grupos foi o tipo de casca. Pela análise dos dados, foi possível determinar que os grupos I, II e III agruparam árvores de fuste com casca lisa, enquanto que o grupo IV agrupou árvores com casca lisa, rugosa e fibrosa, o que o diferenciou dos demais como o grupo mais variável quanto à característica fenotípica tipo de casca.

As duas variáveis relação diâmetro/altura e percentual volumétrico de alburno apresentaram baixo poder de discriminação dos grupos, mas influenciaram na classificação dos mesmos.

A relação diâmetro/altura apresentou distribuição similar ao DAP entre os grupos, ou seja, a maior média pertenceu ao grupo III, enquanto que o grupo I apresentou a menor média para a característica analisada.

O percentual volumétrico de alburno apresentou a maior amplitude de valores no grupo IV, o que possibilita uma seleção de árvores para produção de produtos de madeira tratada com soluções preservativas, como postes, linhas, tramas, etc.

Através da prévia caracterização da madeira, sabe-se que o percentual volumétrico de alburno é inversamente proporcional ao percentual volumétrico de cerne. Considerando isso, o grupo II, que apresentou a menor média de percentual volumétrico de alburno foi o grupo que obteve o maior percentual volumétrico de cerne, apresentando, assim, as árvores com a maior tendência em produzir madeira serrada 
uniforme oriunda da região de cerne e menos indicada à produção de postes, moirões, linhas, etc.

\section{CONCLUSÕES E RECOMENDAÇÕES}

De acordo com a análise dos resultados obtidos, pode-se concluir que:

A variabilidade das características entre as árvores matrizes estudadas possibilitou a realização de ensaios de seleção através de técnicas de agrupamento;

Pela análise de Cluster, as árvores matrizes foram reunidas em quatro grupos distintos, com 88,9\% de probabilidade de serem classificadas corretamente;

Pela análise de discriminante, obtiveram-se cinco variáveis com poder de discriminação e três funções discriminantes. O diâmetro à altura do peito (DAP), a relação altura/diâmetro (RHD), o tipo de casca $(\mathrm{CT})$, o perímetro estimado de rachaduras (PER) e o percentual volumétrico de alburno $\left(\% \mathrm{~V}_{\mathrm{AB}}\right)$ foram as variáveis mais importantes na discriminação dos grupos. Salienta-se que duas variáveis (PER e $\left.\% \mathrm{~V}_{\mathrm{AB}}\right)$ necessitam de métodos destrutivos de determinação;

As árvores do grupo I apresentaram menor tendência em produzir rachaduras de topo das tábuas, enquanto que as dos grupos II e III foram as que mostraram menor tendência em desenvolver rachaduras de topo das toras;

O grupo IV reuniu as árvores matrizes com a maior tendência em produzir rachaduras de topo das toras e das tábuas;

As melhores características dendrométricas e tecnológicas da madeira foram observadas nas árvores do grupo III, o que as classifica como superiores; as características tecnológicas da madeira das árvores do grupo IV não justificam sua seleção e propagação.

Com base nesses resultados, recomenda-se:

Conduzir a brotação das melhores árvores matrizes dos grupos I e III para coleta futura de material;

Avaliar a inclusão de novas variáveis, como as propriedades mecânicas da madeira, na análise de agrupamento das árvores.

\section{AGRADECIMENTOS}

Os autores agradecem à empresa Flosul Indústria e Comércio de Madeiras Ltda, pelo fornecimento da matéria-prima e apoio técnico, e ao servidor Jorge Puhales, pelo auxílio na preparação do material.

\section{REFERÊNCIAS BIBLIOGRÁFICAS}

ARAÚJO, H. J. B. Agrupamento das espécies madeireiras ocorrentes em pequenas áreas sob manejo florestal do projeto de colonização Pedro Peixoto (AC) por similaridade das propriedades físicas e mecânicas. 2002. 184p. Dissertação (Mestrado em Ciências Florestais) - Escola Superior de Agricultura Luiz de Queiroz, Piracicaba, 2002.

ASSIS, T. F. Qualificação tecnológica da madeira de Eucalyptus para serraria: aspectos genéticos e de manejo. In: SIMPÓSIO LATINO-AMERICANO SOBRE MANEJO FLORESTAL, 1., 2000, Santa Maria. Anais... Santa Maria: Programa de Pós-graduação em Engenharia Florestal/UFSM, 2000. p. 59-80.

LONGHI, S. J. Agrupamento e análise fitossociológica de comunidades florestais na sub-bacia hidrográfica do rio Passo Fundo-RS. 1997. 252f. Tese (Doutorado em Ciências Florestais)- Universidade Federal do Paraná, Curitiba, 1997.

LOPES, M.C. Agrupamento de árvores matrizes de eucalyptus grandis em função das variáveis dendrométricas e das características tecnológicas da madeira. 2003. 93f Disssertação (Mestrado em Engenharia Florestal) Universidade Federal de Santa Maria, Santa Maria, 2003.

LOUZADA, J. L. P. C.; MARCOS, S. M. R.; SILVA, M. E. C. M. Avaliação do comportamento de secagem da madeira em estufa para 14 espécies florestais. In: CONGRESSO IBERO-AMERICANO DE PRODUTOS E PESQUISA FLORESTAIS E SEMINÁRIO EM TECNOLOGIA DA MADEIRA E PRODUTOS FLORESTAIS NÃO-MADEIRÁVEIS, 2., 2002, Curitiba. Anais...Curitiba: FUPEF, 2002. 
RUY, O. F.; FERREIRA, M.; TOMAZELLO FILHO, M. Variação da qualidade da madeira entre grupos fenotípicos de clones de Eucalyptus urophylla S.T. Blake da Ilha de Flores, Indonésia. Scientia Forestalis, Piracicaba, n.60, p. 21$27,2001$.

SANTOS, P. E.T.; GERALDI, I. O.; GARCIA, J. N. Estimativas de parâmetros genéticos de propriedades físicas e mecânicas da madeira em eucalyptus grandis. Scientia Forestalis, Piracicaba, n.63, p.54-64, 2003.

XAVIER, A.; BORGES, R. C. G.; CRUZ, C. D. et al. Aplicação da análise multivariada da divergência genética no melhoramento de Eucalyptus spp. Revista Árvore, Viçosa, v.20, n.4, p.495-505, 1996.

ZOBEL, B. J. \& JETT, J. B. Genetics of Wood Production. Syracuse: Springer-Verlag, U.S.A., 1995. 